\title{
Correlações entre caracteres agronômicos em dois ciclos de seleção recorrente em milho-pipoca
}

\author{
Correlations among agronomic traits in two recurrent selection \\ cycles in popcorn
}

\author{
Máskio Daros ${ }^{1}$ Antonio Teixeira do Amaral Júnior ${ }^{3}$ Messias Gonzaga Pereira ${ }^{3}$ \\ Fabrício Santana Santos ${ }^{2}$ Carlos Alberto Scapim ${ }^{4}$ Silvério de Paiva Freitas Júnior ${ }^{2}$ \\ Rogério Figueiredo Daher ${ }^{1}$ Marizangela Rizzatti Ávila ${ }^{4}$
}

\section{RESUMO}

\begin{abstract}
Com o propósito de monitorar o comportamento de blocos gênicos em diferentes gerações de seleção recorrente em milho pipoca, quantificaram-se as correlações fenotípica, genotípica e de ambiente entre caracteres agronômicos, em dois ciclos de seleção recorrente. $O$ primeiro ciclo foi formado por famílias de irmãos completos e o segundo ciclo por famílias $S_{1}$. Embora não significativa, em ambos os ciclos, houve correlação genotípica negativa entre capacidade de expansão e rendimento de grãos. Para o segundo ciclo, as maiores magnitudes de correlação genotípica positiva ocorreram entre rendimento de grãos, número de espigas sadias e estande, indicando a possibilidade de sucesso na seleção de uma ou outra, com base no rendimento de grãos. Embora não significativa e em baixa magnitude, houve acréscimo da correlação genotípica entre rendimento de grãos e capacidade de expansão do primeiro para o segundo ciclo, denotando aumento na concentração de alelos favoráveis na população.
\end{abstract}

Palavras-chave: Zea mays, associação, rendimento, capacidade de expansão.

\section{ABSTRACT}

This work was carried out to estimate environment, genotypic and phenotypic correlations among traits in two intrapopulational recurrent selection cycles in popcorn. The first cycle was composed by full sib families and the second cycle by $S_{1}$ families. Seventy-five families of full sib and two hundred and twenty two families $S$ were evaluated in randomized complete block design with two replicates in two environments. Although not statistically significant, genotypic correlation between grain yield and popping expansion was negative in both selection cycles. For the second cycle of recurrent selection, the highest positive estimates of genotypic correlations were found between: grain yield and number of ears and grain yield and stand, indicating the perspective of the success for selection based on grain yield. Although in less magnitude there was an increase in genotypic correlation between the grain yield and popping expansion from the first to the second cycle of recurrent selection, indicating increase in the favorable allele frequencies in both traits.

Key words: Zea mays, association, grain yield and popping expansion.

\section{INTRODUÇÃO}

Em programas de melhoramento, a eficiência da seleção de determinado caráter pode ser aumentada com a utilização de caracteres agronômicos correlacionados. A prática de seleção em um caráter pode alterar a média de todos os outros com as quais é correlacionado geneticamente, causando efeito indireto de seleção em tais caracteres (VILARINHO, 2001). Isso assume importância relevante, uma vez que o melhorista está interessado na melhoria de um conjunto de caracteres (CRUZ \& REGAZZI, 2001).

Em milho pipoca, por exemplo, dois caracteres são de relevante interesse para o melhoramento de plantas, quais sejam, capacidade de expansão e rendimento de grãos, por atenderem as necessidades do consumidor e do produtor. Todavia,

\footnotetext{
${ }^{1}$ Engenheiro Agrônomo, Doutor em Produção Vegetal, Laboratório de Melhoramento Genético Vegetal, Universidade Estadual do Norte Fluminense Darcy Ribeiro (UENF), 28013-600, Campos dos Goytacazes, RJ.

${ }^{2}$ Engenheiro Agrônomo, Pós-graduando em Produção Vegetal, Laboratório de Melhoramento Genético Vegetal, UENF.

${ }^{3}$ Engenheiro Agrônomo, Doutor, Professor, Laboratório de Melhoramento Genético Vegetal, UENF. Bolsista do Conselho Nacional de Desenvolvimento Científico e Tecnológico (CNPq). E-mail:amaraljr@uenf.br. Autor para correspondência.

${ }^{4}$ Engenheiro Agrônomo, Doutor, Professor, Departamento de Agronomia, Universidade Estadual de Maringá (UEM), Maringá, PR. Bolsista do CNPq.
} 
diversas citações destacam a capacidade de expansão como caráter correlacionado negativamente com outros caracteres agronômicos importantes, como é o caso do rendimento de grãos (BRUNSON, 1937; LIMA et al., 1971; ZINSLY \& MACHADO, 1987; MERLO et al., 1988; DOFING et al., 1991; ANDRADE, 1996). Da mesma forma, CARPENTIERI-PÍPOLO et al. (2002), ao avaliarem sete populações de milho pipoca, encontraram valores de correlação de $-0,2230$ entre capacidade de expansão e rendimento dos grãos. Tais valores, embora tenham sido provenientes da avaliação de populações 'per se', também auxiliam na indicação de que a seleção truncada em apenas um dos caracteres poderá ocasionar variações indesejáveis no outro.

VILARINHO (2001) realizou estudos de correlações entre os ciclos $S_{1}$ e $S_{2}$, de seleção recorrente em milho pipoca e constatou modificações nos valores das associações entre caracteres da planta, da espiga e do grão. Como exemplo, o autor obteve valores de correlação genotípica entre capacidade de expansão e rendimento de grãos, de-0,3900 -0,3100, respectivamente, em relação ao primeiro e segundo ciclos de seleção.

Considerando a necessidade de conhecer o comportamento de blocos gênicos envolvidos na expressão de caracteres de interesse para o melhoramento do milho pipoca nos diferentes ciclos de seleção recorrente, desenvolveu-se o presente trabalho, que teve como objetivo estimar as correlações fenotípicas, genotípicas e de ambiente entre caracteres agronômicos da cultura, em dois ciclos de seleção recorrente e monitorar o comportamento da associação entre a capacidade de expansão e o rendimento de grãos.

\section{MATERIAIS E MÉTODOS}

Dois ciclos de seleção recorrente intrapopulacional de famílias de irmãos completos e famílias endogâmicas foram implementados.

No primeiro ciclo, setenta e cinco famílias de irmãos completos foram cultivadas em outubro de 1999, em blocos ao acaso, com duas repetições dentro de grupos (HALLAUER \& MIRANDA FILHO, 1988), em dois locais, respectivamente, Estações Experimentais da PESAGRO-RIO, de Campos dos Goytacazes e de Itaocara, RJ. Cada grupo conteve 25 famílias. As parcelas foram constituídas por fileiras de $5,0 \mathrm{~m}$ de comprimento, espaçadas em $1,0 \mathrm{~m}$ e com $0,20 \mathrm{~m}$ entre plantas.

Os tratos culturais foram efetuados conforme o recomendado para a cultura (EMBRAPA, 1993; FACELLI \& DOURADO NETO, 2000).
Os seguintes caracteres foram avaliados: rendimento de grãos, em kg.ha ${ }^{-1}$ (PROD); e capacidade de expansão (CE). Para a determinação da capacidade de expansão, foram obtidas amostras ao acaso de cada parcela colhida. O volume, em $\mathrm{cm}^{3}$, de 40 gramas de grãos com cerca de $12 \%$ de umidade foi quantificado antes e após o pipocamento, produzindo a correspondente relação $\mathrm{CE}=$ volume após pipocamento / volume antes do pipocamento. A avaliação da $\mathrm{CE}$ foi realizada em microondas com força máxima de $900 \mathrm{~W}$ por 3 minutos, para cada amostra, sendo que, de cada parcela, obtiveram-se duas amostras.

No segundo ciclo de seleção recorrente, 222 famílias endogâmicas foram avaliadas em março de 2002, em dois locais distintos: Escola Estadual Agrícola Antônio Sarlo, em Campos dos Goytacazes e Estação Experimental da PESAGRO-RIO, em Itaocara, RJ. O delineamento experimental e os tratos culturais foram similares aos adotados no primeiro ciclo de seleção recorrente.

Os seguintes caracteres foram avaliados: estande (ST), expresso pelo número de plantas contidas na fileira; número de plantas quebradas (PLQ), expresso pelo número de plantas que apresentaram o colmo quebrado abaixo da espiga superior, por ocasião da colheita; número de espigas mal empalhadas (EME), obtido pela contagem do número de espigas que não foram cobertas totalmente pela palha; número de espigas sadias (NES), obtido pela contagem do número de espigas colhidas em cada parcela; número de espigas doentes (NED), obtido pela contagem do número de espigas doentes colhidas na parcela; rendimento de grãos (PROD), expresso pelo peso dos grãos após debulha, em g por parcela, e transformado em kg.ha- ${ }^{-1}$; e capacidade de expansão (CE), obtida pela razão entre o volume expandido e o volume inicial de grãos.

A avaliação da capacidade de expansão foi realizada em um pipocador desenvolvido pela EMPRAPA - Centro Nacional de Pesquisa e Desenvolvimento de Instrumentação Agropecuária (CNPDIA) com resistência elétrica e termostato. Para tanto, obtiveram-se de cada família, duas amostras de $30 \mathrm{~mL}$ de grãos. Cada amostra foi colocada no medidor de expansão por um tempo de 2,5 minutos, após este ter atingido a temperatura de $270^{\circ} \mathrm{C}$. O volume expandido foi quantificado numa proveta graduada de $1.000 \mathrm{~mL}$.

As estimativas dos coeficientes de correlação fenotípica $\left(\mathrm{r}_{\mathrm{F}}\right)$ genotípica $\left(\mathrm{r}_{\mathrm{G}}\right)$ e de ambiente $\left(\mathrm{r}_{\mathrm{A}}\right)$ entre os pares de caracteres nos dois ciclos de seleção recorrente foram obtidas conforme CRUZ \& 
REGAZZI (2001). A significância dos coeficientes de correlação foi avaliada pelo teste t, em $5 \%$ e $1 \%$ de probabilidade de erro (STEEL \& TORRIE, 1980).

\section{RESULTADOS E DISCUSSÃO}

As estimativas das correlações fenotípicas $\left(\mathrm{r}_{\mathrm{F}}\right)$, genotípicas $\left(\mathrm{r}_{\mathrm{G}}\right)$ e de ambiente $\left(\mathrm{r}_{\mathrm{A}}\right)$ entre os caracteres capacidade de expansão e rendimento de grãos, no primeiro ciclo de seleção foram, respectivamente, $-0,1648,-0,2648$ e 0,0371 . Nenhum desses valores foi significativo pelo teste ' $t$ ', a $5 \%$ de probabilidade de erro. Essas magnitudes, quando comparadas com as obtidas para os mesmos caracteres no segundo ciclo de seleção (Tabela 1), denotam que houve tendência de alteração de correlação, em razão dos valores expressos para a correlação genotípica serem, respectivamente, $-0,2648$ e $-0,0643$, para o primeiro e segundo ciclos. Todavia, essa consideração deve ser concebida tão somente como um indício, uma vez que as magnitudes das correlações, por não serem significativas, devem ser consideradas, estatisticamente, iguais a zero.

Com relação à tabela 2 , constata-se que reduzida proporção de pares de caracteres $(19,00 \%)$ expressou as correlações genotípicas maiores que as fenotípicas e de ambiente, indicando que o componente de ambiente teve influência na expressão dos caracteres.

Com base no segundo ciclo de seleção, sete pares de caracteres apresentaram correlações genotípicas significativas, sendo que cinco foram positivas (Tabela 2). Correlações genotípicas positivas indicam que a seleção objetivando aumento de um deles provocará o mesmo efeito no outro. Adotando-se como exemplo as maiores magnitudes de correlações genotípicas expressas, pode-se afirmar que maiores ganhos via seleção indireta são possíveis entre rendimento de grãos, número de espigas sadias e estande.

Conforme a tabela 2, todos os caracteres avaliados expressaram correlação não significativa com capacidade de expansão, exceto o número de espigas mal empalhadas e o rendimento de grãos. Em particular ao número de espigas mal empalhadas, este revelou correlação genotípica negativa com capacidade de expansão, evidenciando que grãos expostos às intempéries antes da colheita têm o pericarpo danificado e, como conseqüência, diminuição da capacidade de expansão. A mesma afirmação foi feita por HOSENEY et al. (1983). Segundo estes, qualquer dano no pericarpo provoca acentuada queda na capacidade de expansão. Vale

Tabela 1 - Estimativas dos coeficientes de correlação fenotípica $\left(\mathrm{r}_{\mathrm{F}}\right)$, genotípica $\left(\mathrm{r}_{\mathrm{G}}\right)$ e de ambiente $\left(\mathrm{r}_{\mathrm{A}}\right)$ entre capacidade de expansão $(\mathrm{CE})$, estande (ST), número de plantas quebradas (PLQ), número de espigas mal empalhadas (EME), número de espigas sadias (NES), número de espigas doentes (NED) e rendimento de grãos (PROD), avaliadas em duzentas e vinte e duas famílias endogâmicas de milho pipoca, na análise conjunta dos ambientes Campos dos Goytacazes e Itaocara, RJ.

\begin{tabular}{|c|c|c|c|c|c|c|}
\hline Caráter ${ }^{1 /}$ & ST & PLQ & EME & NES & NED & PROD \\
\hline \multirow{3}{*}{$\mathrm{CE}$} & $0,0633 \mathrm{~ns}$ & $-0,0611 \mathrm{~ns}$ & $-0,0531 \mathrm{~ns}$ & $0,0220 \mathrm{~ns}$ & $-0,0733 \mathrm{~ns}$ & $0,0571 \mathrm{~ns}$ \\
\hline & $0,0159 \mathrm{~ns}$ & $-0,0553 \mathrm{~ns}$ & $-0,1543^{*}$ & $-0,0082 \mathrm{~ns}$ & $-0,1119 \mathrm{~ns}$ & $-0,0643 \mathrm{~ns}$ \\
\hline & $0,1286 \mathrm{~ns}$ & $-0,1104 \mathrm{~ns}$ & $0,0271 \mathrm{~ns}$ & $0,0720 \mathrm{~ns}$ & $-0,0560 \mathrm{~ns}$ & $0,1857 * *$ \\
\hline \multirow{3}{*}{ ST } & & $0,1000 \mathrm{~ns}$ & $0,0377 \mathrm{~ns}$ & $0,2530^{* *}$ & $0,1665^{*}$ & $0,2458^{* *}$ \\
\hline & & $0,0091 \mathrm{~ns}$ & $-0,0103 \mathrm{~ns}$ & $0,2958 * *$ & $0,0727 \mathrm{~ns}$ & $0,3098 * *$ \\
\hline & & $0,1640^{*}$ & $0,0795 \mathrm{~ns}$ & $0,3211^{* *}$ & $0,1818^{* *}$ & $0,2977 * *$ \\
\hline \multirow{3}{*}{ PLQ } & & & $0,1481 \mathrm{~ns}$ & $-0,1493 \mathrm{~ns}$ & $0,1541 *$ & $-0,1209 \mathrm{~ns}$ \\
\hline & & & $0,0048 \mathrm{~ns}$ & $-0,0058 \mathrm{~ns}$ & $0,1174 \mathrm{~ns}$ & $-0,0459 \mathrm{~ns}$ \\
\hline & & & $0,0622 \mathrm{~ns}$ & $-0,0554 \mathrm{~ns}$ & $0,1232 \mathrm{~ns}$ & $-0,0650 \mathrm{~ns}$ \\
\hline \multirow{3}{*}{ EME } & & & & $-0,0982 \mathrm{~ns}$ & $0,1599 *$ & $0,0363 \mathrm{~ns}$ \\
\hline & & & & $-0,0404 \mathrm{~ns}$ & $0,2183^{* *}$ & $0,1607^{*}$ \\
\hline & & & & $-0,0105 \mathrm{~ns}$ & $0,0176 \mathrm{~ns}$ & $-0,0122 \mathrm{~ns}$ \\
\hline \multirow{3}{*}{ NES } & & & & & $-0,2208^{* *}$ & $0,8034 * *$ \\
\hline & & & & & $-0,1807 * *$ & $0,8078^{* *}$ \\
\hline & & & & & $-0,2329 * *$ & $0,8132 * *$ \\
\hline \multirow{3}{*}{ NED } & & & & & & $-0,1572^{*}$ \\
\hline & & & & & & $-0,1119 \mathrm{~ns}$ \\
\hline & & & & & & $-0,1808^{* *}$ \\
\hline
\end{tabular}

* = significativo pelo teste 't' em nível de $5 \%$ de probabilidade de erro; ns = não significativo.

Ciência Rural, v.34, n.5, set-out, 2004. 
Tabela 2 - Estimativas dos coeficientes de correlação fenotípica $\left(\mathrm{r}_{\mathrm{F}}\right)$, genotípica $\left(\mathrm{r}_{\mathrm{G}}\right)$ e de ambiente $\left(\mathrm{r}_{\mathrm{A}}\right)$ entre capacidade de expansão (CE), estande (ST), número de plantas quebradas (PLQ), número de espigas mal empalhadas (EME), número de espigas sadias (NES), número de espigas doentes (NED) e rendimento de grãos (PROD), avaliadas em duzentas e vinte e duas famílias endogâmicas de milho pipoca em Campos dos Goytacazes, RJ.

\begin{tabular}{|c|c|c|c|c|c|c|c|}
\hline Caráter ${ }^{1 /}$ & $\mathrm{r}$ & ST & PLQ & EME & NES & NED & PROD \\
\hline \multirow{3}{*}{$\mathrm{CE}$} & $\mathrm{r}_{\mathrm{F}}$ & $0,0609 \mathrm{~ns}$ & $-0,0619 \mathrm{~ns}$ & $-0,0509 \mathrm{~ns}$ & $0,0729 \mathrm{~ns}$ & $-0,0358 \mathrm{~ns}$ & $0,1502 *$ \\
\hline & $r_{G}$ & $0,0511 \mathrm{~ns}$ & $-0,0342 \mathrm{~ns}$ & $-0,0662 \mathrm{~ns}$ & $0,0951 \mathrm{~ns}$ & $-0,0781 \mathrm{~ns}$ & $0,1181 \mathrm{~ns}$ \\
\hline & $\mathrm{r}_{\mathrm{A}}$ & $-0,002 \mathrm{~ns}$ & $-0,1292 \mathrm{~ns}$ & $0,0606 \mathrm{~ns}$ & $-0,0209 \mathrm{~ns}$ & $-0,0031 \mathrm{~ns}$ & $0,1796 * *$ \\
\hline \multirow{3}{*}{ ST } & $\mathrm{r}_{\mathrm{F}}$ & & $0,2436^{* *}$ & $0,0257 \mathrm{~ns}$ & $0,2149 * *$ & $0,2264 * *$ & $0,2018 * *$ \\
\hline & $\mathrm{r}_{\mathrm{G}}$ & & $0,2142 * *$ & $0,0270 \mathrm{~ns}$ & $0,2773 * *$ & $0,1684 * *$ & $0,2527 * *$ \\
\hline & $\mathrm{r}_{\mathrm{A}}$ & & $0,3543 * *$ & $0,1240 \mathrm{~ns}$ & $0,2119 * *$ & $0,2882 * *$ & $0,2067 * *$ \\
\hline \multirow{3}{*}{ PLQ } & $\mathrm{r}_{\mathrm{F}}$ & & & $0,0685 \mathrm{~ns}$ & $-0,1797 * *$ & $0,1786 * *$ & $-0,2187 * *$ \\
\hline & $\mathrm{r}_{\mathrm{G}}$ & & & $0,0501 \mathrm{~ns}$ & $-0,2329 * *$ & $0,1709 * *$ & $-0,2686 * *$ \\
\hline & $\mathrm{r}_{\mathrm{A}}$ & & & $0,1205 \mathrm{~ns}$ & $-0,0276 \mathrm{~ns}$ & $0,1720^{* *}$ & $-0,0530 \mathrm{~ns}$ \\
\hline \multirow{3}{*}{ EME } & $\mathrm{r}_{\mathrm{F}}$ & & & & $-0,0160 \mathrm{~ns}$ & $0,0863 \mathrm{~ns}$ & $0,1184 \mathrm{~ns}$ \\
\hline & $\mathrm{r}_{\mathrm{G}}$ & & & & $-0,0163 \mathrm{~ns}$ & $0,1354 \mathrm{~ns}$ & $0,1683 * *$ \\
\hline & $\mathrm{r}_{\mathrm{A}}$ & & & & $0,0285 \mathrm{~ns}$ & $0,0537 \mathrm{~ns}$ & $0,0294 \mathrm{~ns}$ \\
\hline \multirow{3}{*}{ NES } & $\mathrm{r}_{\mathrm{F}}$ & & & & & $-0,2371^{* *}$ & $0,7608^{* *}$ \\
\hline & $\mathrm{r}_{\mathrm{G}}$ & & & & & $-0,1992^{* *}$ & $0,7672 * *$ \\
\hline & $\mathrm{r}_{\mathrm{A}}$ & & & & & $-0,3246 * *$ & $0,7528 * *$ \\
\hline \multirow{3}{*}{ NED } & $\mathrm{r}_{\mathrm{F}}$ & & & & & & $-0,1944 * *$ \\
\hline & $\mathrm{r}_{\mathrm{G}}$ & & & & & & $-0,1472 * *$ \\
\hline & $r_{A}$ & & & & & & $-0,3104 * *$ \\
\hline
\end{tabular}

* = significativo pelo teste 't' em nível de 5\% de probabilidade de erro; ns = não significativo.

ressaltar que essa estimativa é significativa, no entanto, de baixo valor.

Estimativa de correlação genotípica positiva, no entanto, estatisticamente igual a zero, ocorreu entre os caracteres capacidade de expansão e rendimento de grãos (Tabela 2), evidenciando que é possível em um programa de melhoramento de milho pipoca, a seleção de cultivares com efeito positivo para ambos os caracteres. Resultados concordantes foram enunciados por LINARES (1987) e SAWAZAKI (1996).

Para a obtenção de ganhos superiores para rendimento de grãos e capacidade de produção, a utilização de índices de seleção é um procedimento hábil, que deve ser considerado em programas de melhoramento com milho pipoca. Apesar de enfatizada a potencialidade dos índices de seleção no melhoramento de plantas (CRUZ \& REGAZZI, 2001), em milho pipoca, apenas mais recentemente alguns trabalhos têm empregado a metodologia com adequada propriedade, como por exemplo, VILARINHO (2001), GRANATE et al. (2002) e SANTOS et al. (2003).

Estimativas de correlações de ambiente significativas e negativas ocorreram entre os caracteres número de espigas sadias e número de espigas doentes e entre número de espigas doentes e rendimento de grãos (Tabela 2). Esses resultados traduzem o efeito da incidência de doenças para com o rendimento de grãos. Não apenas a ocorrência de doenças, mas também caracteres da espiga têm implicação direta no rendimento de grãos, o que pode ser verificado, no presente trabalho, pela associação significativa entre número de espigas doentes e número de espigas mal empalhadas, embora tal associação tenha sido expressa por baixa magnitude (Tabela 2). Isto se deve ao fato de que o número de espigas mal empalhadas é associado, genotipicamente, com significância em 5 $\%$ de probabilidade de erro, pelo teste ' $t$ ', com rendimento de grãos (Tabela 2).

Embora seja possível fazer inferências das correlações provenientes da análise conjunta dos locais (Tabela 2), algumas diferenças são perceptíveis quando se comparam esses resultados com os obtidos nas análises dos locais em separado (Tabelas 1 e 3 ). O caráter número de plantas quebradas, por exemplo, apresentou correlação genotípica próxima de zero para com os demais caracteres na análise conjunta (Tabela 2) e no local Itaocara (Tabela 3). Já, em Campos dos Goytacazes, o número de plantas quebradas revelou correlação genotípica altamente significativa para com os caracteres número de espigas doentes, número de espigas sadias e rendimento de grãos (Tabela 1), o que provavelmente se deveu à maior intensidade dos ventos que naturalmente ocorrem nesta localidade. 
Ao comparar a relação entre os caracteres número de espigas mal empalhadas, número de espigas doentes e rendimento de grãos, percebe-se comportamento inverso nos locais estudados no segundo ciclo de seleção recorrente (Tabelas 1 e 3 ). Por esta análise, verifica-se que número de espigas mal empalhadas revelou correlação genotípica não significativa para com número de espigas doentes, mas altamente significativa para com rendimento de grãos, em Campos dos Goytacazes (Tabela 1). Por sua vez, em Itaocara, número de espigas mal empalhadas expressou correlação genotípica altamente significativa para com número de espigas doentes e não significativa para com rendimento de grãos (Tabela 3 ).

Isso denota que, a despeito da elevada correlação entre os locais, nos dois ciclos de seleção recorrente; para o segundo ciclo, a localidade de Campos dos Goytacazes possibilitou a obtenção de materiais com melhor empalhamento e rendimento de grãos e, não obstante, com maior capacidade de expansão. Essa última associação é ratificada pela significância, em $5 \%$ de probabilidade de erro, pelo teste 't', para a correlação fenotípica entre CE e PROD (Tabela 1).

Porém, não houve inversão de sinal nas correlações genotípicas entre número de espigas mal empalhadas, número de espigas doentes e rendimento de grãos, nos locais avaliados no segundo ciclo de seleção (Tabelas 2 e 3 ), o que denota que as diferenças de ambiente não foram suficientemente consideráveis para sustentar o argumento da necessidade de estabelecimento de programas de melhoramento próprios para cada ambiente. Isso posto, um único programa de seleção recorrente para o Norte e Noroeste Fluminense poderá promover o acúmulo de alelos favoráveis na população em seleção, o que implica economia de tempo, esforço e recurso.

\section{CONCLUSÃO}

Há uma tendência de acréscimo da correlação genotípica entre rendimento de grãos e capacidade de expansão do primeiro para o segundo ciclo de seleção recorrente, indicando um aumento na concentração de alelos favoráveis.

Tabela 3 - Estimativas dos coeficientes de correlação fenotípica $\left(\mathrm{r}_{\mathrm{F}}\right)$, genotípica $\left(\mathrm{r}_{\mathrm{G}}\right)$ e de ambiente $\left(\mathrm{r}_{\mathrm{A}}\right)$ entre capacidade de expansão $(\mathrm{CE})$, estande (ST), número de plantas quebradas (PLQ), número de espigas mal empalhadas (EME), número de espigas sadias (NES), número de espigas doentes (NED) e rendimento de grãos (PROD), avaliadas em duzentas e vinte e duas famílias endogâmicas de milho pipoca em Itaocara, RJ.

\begin{tabular}{|c|c|c|c|c|c|c|c|}
\hline Caráter $^{1 /}$ & $\mathrm{r}$ & ST & PLQ & EME & NES & NED & PROD \\
\hline \multirow{3}{*}{$\mathrm{CE}$} & $\mathrm{r}_{\mathrm{F}}$ & $0,0625 \mathrm{~ns}$ & $-0,0933 \mathrm{~ns}$ & $-0,1419 *$ & $-0,0052 \mathrm{~ns}$ & $-0,1271 \mathrm{~ns}$ & $-0,0143 \mathrm{~ns}$ \\
\hline & $\mathrm{r}_{\mathrm{G}}$ & $-0,0001 \mathrm{~ns}$ & $-0,1093 \mathrm{~ns}$ & $-0,1701 * *$ & $-0,0518 \mathrm{~ns}$ & $-0,1247 \mathrm{~ns}$ & $-0,0825 \mathrm{~ns}$ \\
\hline & $\mathrm{r}_{\mathrm{A}}$ & $0,2531 * *$ & $-0,0901 \mathrm{~ns}$ & $-0,0412 \mathrm{~ns}$ & $-0,1394^{*}$ & $-0,1130 \mathrm{~ns}$ & $0,1929 * *$ \\
\hline \multirow{3}{*}{ ST } & $\mathrm{r}_{\mathrm{F}}$ & & $-0,1007 \mathrm{~ns}$ & $-0,0148 \mathrm{~ns}$ & $0,3234 * *$ & $0,0863 \mathrm{~ns}$ & $0,2879 * *$ \\
\hline & $\mathrm{r}_{\mathrm{G}}$ & & $-0,1413^{*}$ & $-0,0801 \mathrm{~ns}$ & $0,2419 * *$ & $0,0467 \mathrm{~ns}$ & $0,2357 * *$ \\
\hline & $\mathrm{r}_{\mathrm{A}}$ & & $-0,0737 \mathrm{~ns}$ & $0,0062 \mathrm{~ns}$ & $0,4094 * *$ & $0,0626 \mathrm{~ns}$ & $0,3709 * *$ \\
\hline \multirow{3}{*}{ PLQ } & $\mathrm{r}_{\mathrm{F}}$ & & & $-0,1057 \mathrm{~ns}$ & $0,0078 \mathrm{~ns}$ & $-0,0138 \mathrm{~ns}$ & $-0,0131 \mathrm{~ns}$ \\
\hline & $\mathrm{r}_{\mathrm{G}}$ & & & $-0,0678 \mathrm{~ns}$ & $0,0246 \mathrm{~ns}$ & $0,0058 \mathrm{~ns}$ & $-0,0240 \mathrm{~ns}$ \\
\hline & $\mathrm{r}_{\mathrm{A}}$ & & & $-0,1018 \mathrm{~ns}$ & $-0,0849 \mathrm{~ns}$ & $0,0556 \mathrm{~ns}$ & $-0,0806 \mathrm{~ns}$ \\
\hline \multirow{3}{*}{ EME } & $\mathrm{r}_{\mathrm{F}}$ & & & & $-0,0612 \mathrm{~ns}$ & $0,1938^{* *} *$ & $-0,0404 \mathrm{~ns}$ \\
\hline & $\mathrm{r}_{\mathrm{G}}$ & & & & $-0,0777 \mathrm{~ns}$ & $0,3434 * *$ & $0,0182 \mathrm{~ns}$ \\
\hline & $\mathrm{r}_{\mathrm{A}}$ & & & & $-0,0848 \mathrm{~ns}$ & $-0,0747 \mathrm{~ns}$ & $-0,0896 \mathrm{~ns}$ \\
\hline \multirow{3}{*}{ NES } & $\mathrm{r}_{\mathrm{F}}$ & & & & & $-0,1505^{*}$ & $0,8461 * *$ \\
\hline & $\mathrm{r}_{\mathrm{G}}$ & & & & & $-0,1985^{* *}$ & $0,8541 * *$ \\
\hline & $\mathrm{r}_{\mathrm{A}}$ & & & & & $-0,1652 * *$ & $0,8458 * *$ \\
\hline \multirow{3}{*}{ NED } & $\mathrm{r}_{\mathrm{F}}$ & & & & & & $-0,1165 n s$ \\
\hline & $\mathrm{r}_{\mathrm{G}}$ & & & & & & $-0,1263 n s$ \\
\hline & $\mathrm{r}_{\mathrm{A}}$ & & & & & & $-0,0843 \mathrm{~ns}$ \\
\hline
\end{tabular}

* = significativo pelo teste 't' em nível de $5 \%$ de probabilidade de erro; ns = não significativo.

Ciência Rural, v.34, n.5, set-out, 2004. 


\section{REFERÊNCIAS BIBLIOGRÁFICAS}

ANDRADE, R.A. Cruzamentos dialélicos entre seis variedades de milho pipoca. 1996. 79f. Dissertação (Mestrado em Genética e Melhoramento) - Curso de Pós-graduação em Genética e Melhoramento, Universidade Federal de Viçosa.

BRUNSON, A.M. Popcorn breeding. Washington: Yearbook Agricultural, v.1, p.395-404, 1937.

CARPENTIERI-PÍPOLO, V. et al. Correlações entre caracteres quantitativos em milho pipoca. Horticultura Brasileira, Brasília, v.20, n.4, p.551$554,2002$.

CRUZ, C.D.; REGAZZI, A.J. Modelos biométricos aplicados ao melhoramento genético. Viçosa : UFV, 2001. 390p.

DOFING, S.M.; D'CROZ-MASON, N.; THOMASCOMPTON, M.A. Inheritance of expansion volume and yield in two popcorn $x$ dent corn crosses. Crop Science, Madison, v.31, p.715-718, 1991.

EMPRESA BRASILEIRA DE PESQUISA AGROPECUÁRIA. Centro Nacional de Pesquisa Milho. Recomendações técnicas para o cultivo do milho. Brasília, 1993. 204p.

FACELli, A.L.; DOURAdo NETO, D. Produção de milho. Guaíba : Agropecuária, 2000. 360p.

GRANATE, M.J.; CRUZ, C.D.; PACHECO, C.A.P. Predição de ganho genético com diferentes índices de seleção no milho pipoca CMS-43. Pesquisa Agropecuária Brasileira, Brasília, v.37, n.7, p.101$108,2002$.

HALLAUER, A.R.; MIRANDA FILHO, J.B. Quantitative genetics in maize breeding. Ames : Iowa State University, 1981. 468p.

HOSENEY, R.C.; ZELEZNAK, K.; ABDELRAHMAN, A. Mechanism of popcorn popping. Journal Cereal Science, London: v.1, p.43-52, 1983.
LIMA, M. et al. Resultados parciais de um programa de melhoramento do milho (Zea mays L.) visando o aumento da produtividade, caracteres agronômicos e capacidade de expansão. Relatório Científico do Departamento e Instituto de Genética, Escola Superior de Agricultura Luiz de Queirós, v.5, p.84-93, 1971.

LINARES, E. Seleção recorrente recíproca em famílias de meio-irmãos em milho pipoca (Zea mays L.). 1987. $78 \mathrm{f}$. Dissertação (Mestrado em Genética e Melhoramento). - Curso de Pós-graduação em Genética e Melhoramento, Escola Superior de Agricultura Luiz de Queiroz-ESALQ.

MERLO, E.; FORNASIERI FILHO, D.; LAM-SÁNCHEZ, A. Avaliação de sete cultivares de milho pipoca (Zea mays, L.) em três densidades de semeadura. Científica, Jaboticabal, v.16, n.2, p.245-251, 1988.

PACHECO, C.A.P. et al. Estimativas de parâmetros genéticos nas populações CMS-42 e CMS-43 de milho pipoca. Pesquisa Agropecuária Brasileira, Brasília, v.33, n.12, p.1995-2001, 1998.

SANTOS, F.S.S. et al. Uso do índice de seleção de Smith \& Hazel na população de milho pipoca UNB-2U para obtenção do segundo ciclo de seleção recorrente. In: CONGRESSO BRASILEIRO DE MELHORAMENTO DE PLANTAS, 2003, Porto Seguro, BA. Anais... Porto Seguro : Brazilian Society of Plant Breeding, 2003. V.2. p.5-5.

SAWAZAKI, E. Parâmetros genéticos em milho pipoca. 1996. 157f. Tese (Doutorado em Genética e Melhoramento) - Curso de Pós-graduação em Genética e Melhoramento, Escola Superior de Agricultura Luiz de Queiroz-ESALQ.

STEEL, R.GD.; TORRIE, J.H. Principles and procedures of statistics: a biometrical approach. New York : McGraw-Hill Book, 1980. 633p.

VILARINHO, A.A. Seleção de progênies endogâmicas $S_{1}$ e $S_{2}$ em programas de melhoramento intrapopulacional e de produção de híbridos de milho pipoca (Zea mays L.). 2001. 79f. Dissertação (Mestrado em Genética e Melhoramento) - Curso de Pós-graduação em Genética e Melhoramento, Universidade Federal de Viçosa.

ZINSLY, J.R.; MACHADO, J.A. Milho pipoca. In: PATERNIANI, E.; VIEGAS, G.P. (Ed). Melhoramento e produção do milho. Campinas : Fundação Cargill, 1987. p.413-421. 